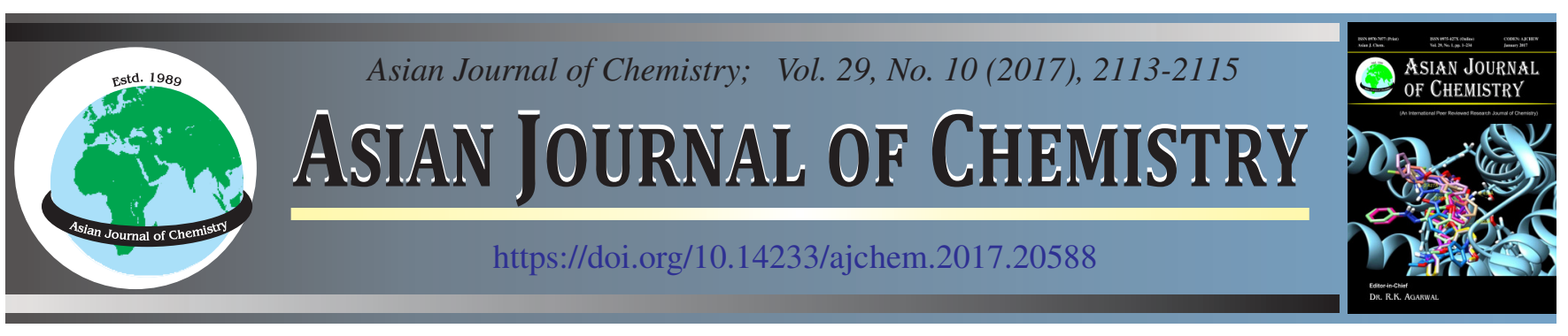

\title{
Design and Synthesis of Antimicrobial Active New Molecular Entities of N-Substituted Pipradol Derivatives
}

\author{
B. Sri Ramudu ${ }^{1, *}$, D. Ramachandran ${ }^{1}$, S. Venkat RaO ${ }^{2}$, Ch. Murali Krishna ${ }^{2}$, \\ B. Satya Narayana ${ }^{2}$ and Kallam NaveEn RedDy ${ }^{2}$
}

${ }^{1}$ Acharya Nagarjuna University, Nagarjuna Nagar, Guntur-522 510, India

${ }^{2}$ Srigen Life Sciences Pvt. Ltd., Plot No. 34, 35, I.E. Rajiv Gandhi Nagar, IDA Kukatpally-500 037, India

*Corresponding author: E-mail: sriram.elm.chem@gmail.com

Received: 15 February 2017;

Accepted: 19 May 2017;

Published online: 31 August 2017;

AJC-18501

Synthesis of antimicrobial 4-(hydroxyldiphenyl methyl)piperidin-1-yl)(substituted phenyl)methanone derivatives by using conventional
chemical reactions to produces feasible and entirely new chemical entities (NCE'S) which were having a great potential microbial
activities equivalent to fexofenidine used as a biological standard. This invention may help full for derive more potential pipradol molecules
with peptide bond linkage.
Keywords: Pipradol derivatives, Antimicrobial, Peptide bond, Fexofenadine.

\section{INTRODUCTION}

In this millennium, the most important drug development areas are antiviral, antifungal or antimicrobial, antidiabetes mellitus, anticancerous, as per as our research budget is concern here we developed some new pipradol derivatives by using simple conventional and economic reagents to generate the new chemical entities with the pipradol having a peptide (-HN$\mathrm{C}=\mathrm{O}$ ) bond linkage. Literature survey revealed that pipecolic acid (piperidine-2-carboxylic acid) is a precursor of various bioactive compounds such as thioridazine [1] (antituberculosis agent), pipradol [2,3] (anticonvulsant agent) which are derived from piperidine-4-carboxylic acid.

Few reports have been appeared on central stimulant activity of various diaryl piperidines, diaryl piperidine methanol [4], few reports available on optical isomers [5,6] and their activities of pipradols [7]. Pipradols N-alkylation's and their drug applications were reported [8-15], however, none of the literature is available for $\mathrm{N}$-acylation i.e., peptide bond formation with $\gamma$ pipradol.

Herein the present work, we have developed some contemporary pipradol with peptide linkage, having chemical name of (4-(hydroxyldiphenyl methyl)piperidin-1-yl) (substituted phenyl)methanone. These analogues synthesized from commercially available raw material diphenyl(piperidin-4-yl)methanol (azacyclonol), which is a basic scaffold in fexofenadine and these molecular derivatives synthesized have more potential towards antimicrobial activity [3] against Candida albicans,
Aspergillus niger and Saccharomyces cerrevisiae with respect to fexofenidine hydrochloride taken as a standard.

\section{EXPERIMENTAL}

All the key raw materials, reagents and solvents were purchased from Aldrich. Instruments used for their analytical testing are ${ }^{1} \mathrm{H}$ NMR (Bruker $400 \mathrm{MHZ}$ ), Mass (Bruker), HPLC (Shimadzu, LC-20 solutions) and IR by Perkin Elmer.

Synthesis of diphenyl(piperidin-4-yl)methanol (4c): In a $3 \mathrm{~L}$ round bottom flask equipped with mechanical stirrer and a water bath the following materials are poured: magnesium turnings ( $100 \mathrm{~g}, 4.16 \mathrm{mmol})$, tetrahydrofuran $(650 \mathrm{~mL})$, bromo benzene ( $43.6 \mathrm{~g}, 0.277 \mathrm{mmol}$ ). The mixture is heated to reflux while stirring and as soon as the reaction is started a solution of brombenzene $(608.4 \mathrm{~g}, 3.87 \mathrm{mmol})$ is slowly added at such a rate so as to maintain reflux. When the addition is complete, the mixture is refluxed until magnesium is almost completely dissolved. Then isonipecotic acid (4a) (200 g) and toluene $(300 \mathrm{~mL})$ are added to phenyl magnesium bromide $(\mathbf{4 b})$ (Grignard reagent) kept at $50{ }^{\circ} \mathrm{C}$. The mixture is refluxed at atmospheric pressure for about $1 \mathrm{~h}$. Then, the reaction is kept under pressure with nitrogen ( 0.4 to $1.5 \mathrm{~atm}$ ) and the mixture is heated again between 105 and $120^{\circ} \mathrm{C}$. for $10 \mathrm{~h}$. After this, the reaction mixture is allowed to cool, the pressure of the reaction is slowly decreased and the mixture is slowly poured into $4 \mathrm{~L}$ of cold water. Yield $415 \mathrm{~g}$ of the azacyclonol [16] compound $\mathbf{4 c}$ of the title (Scheme-I). 


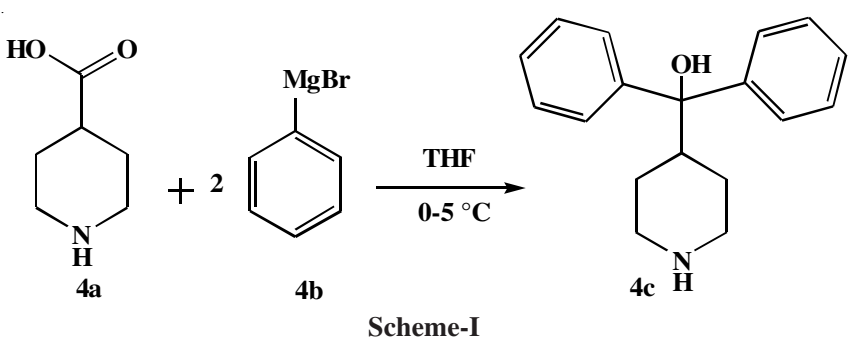

Synthetic method of acid chloride of substituted benzoic acids (2b.a-2b.f): In a $100 \mathrm{~mL}$ round bottom flask equipped with mechanical stirrer and a water bath, taken 2-methyl-3nitrobenzoic acid (15 g, $0.00 \mathrm{mmol})$, chloroform $(200 \mathrm{~mL})$ and then freshly distilled thionyl chloride $(30 \mathrm{~mL})$ was added drop wise at room temperature. The reaction mass stirred for $10 \mathrm{~min}$ at ambient temperature and slowly heated to $70{ }^{\circ} \mathrm{C}$, maintain the same temperature for 1-2 h. After completion of the TLC removed the excess thionyl chloride and once stripped off with chloroform to get desired substituted benzoyl chlorides.

Synthesis of (4-(hydroxydiphenylmethyl)piperidin-1yl)(2-methyl-3-nitrophenyl)methanone (5e): To a $250 \mathrm{~mL}$ round bottom flask, diphenyl(piperidin-4-yl)methanol (12 g), triethylamine $(2 \mathrm{~mL})$ in acetone $(100 \mathrm{~mL})$ under constant stirring were added follwed by the addition of 2-methyl-3-nitrobenzoyl chloride ( $15 \mathrm{~g})$ dropwise at room temperature and then refluxed for 3-4 h. After TLC complies stripped off the acetone and the obtained crude product is subjected to column purification in a mobile phase (1:9) methanol:chloroform. The yellow coloured obtained product yield $15 \mathrm{~g}$ (Scheme-II). m.p.: $140-145{ }^{\circ} \mathrm{C}$. ${ }^{1} \mathrm{H}$ NMR: $\delta 7.8 \mathrm{~d}, 1 \mathrm{H}, \delta 7.45 \mathrm{~d}, 2 \mathrm{H}, \delta 7.40 \mathrm{t}, 3 \mathrm{H}, \delta 7.33 \mathrm{~d}, 4 \mathrm{H}$, $\delta 7.2 \mathrm{~m}, 2 \mathrm{H}, \delta 7.17 \mathrm{t}, 2 \mathrm{H}, \delta 4.85 \mathrm{t}, 1 \mathrm{H}, \delta 3.36 \mathrm{~d}, 1 \mathrm{H}, \delta 3.02 \mathrm{~m}$, $1 \mathrm{H}, \delta 2.8 \mathrm{t}, 1 \mathrm{H}, \delta 2.65 \mathrm{~m}, 1 \mathrm{H}, \delta 2.41 \mathrm{~d}, 3 \mathrm{H}, \delta 2.1 \mathrm{~s}, 2 \mathrm{H}, \delta 1.72 \mathrm{~d}$, $1 \mathrm{H}, \delta 1.53 \mathrm{~s}, 2 \mathrm{H}, \delta 1.4 \mathrm{~m}, 3 \mathrm{H}, \delta 1.29 \mathrm{~m}, 1 \mathrm{H}$. Mass: M: 430.5, $\mathrm{M}+\mathrm{H}: 431$.

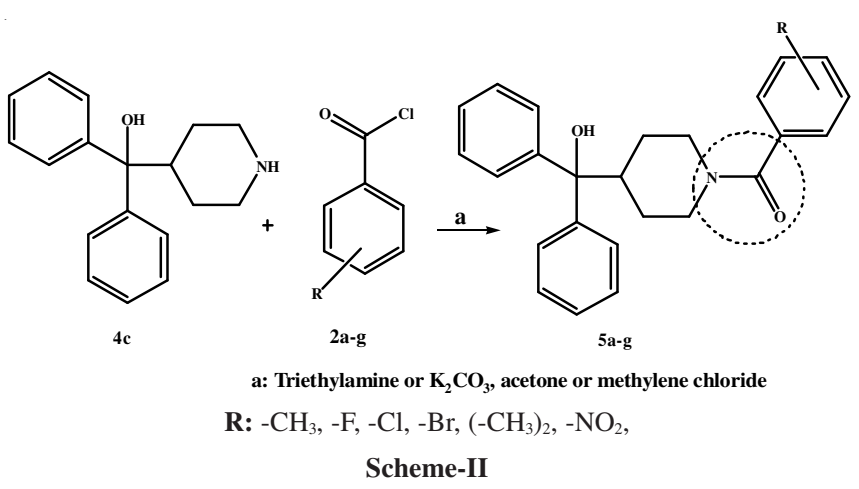

And rest of the compounds also followed the same procedure what we done for compound $\mathbf{5 e}$, hence we incorporated their spectral data for each compound as follows:

Synthesis of (4-(hydroxyl diphenyl methyl)piperidin1-yl)(2,4,5-triflourophenyl)methanone (5a): ${ }^{1} \mathrm{H}$ NMR: $\delta 7.5$ $\mathrm{m}, 4 \mathrm{H}, \delta 7.35 \mathrm{~m}, 5 \mathrm{H}, \delta 7.22 \mathrm{~m}, 2 \mathrm{H}, \delta 609 \mathrm{~m}, 1 \mathrm{H}, \delta 4.74$ $\mathrm{dd}, 1 \mathrm{H}, \delta 3.51 \mathrm{dd}, 1 \mathrm{H}, \delta 3.12 \mathrm{~s}, 1 \mathrm{H}, \delta 2.84 \mathrm{t}, 1 \mathrm{H}, \delta 2.6 \mathrm{t}, 1 \mathrm{H}, \delta$ 1.7 m,1H, $\delta 1.4$ m, 3H. Mass: M: 425.44, M+H: 426.

Synthesis of (4-(hydroxyl diphenyl methyl)piperidin1-yl)(3, 5-dinitrophenyl)methanone (5b): ${ }^{1} \mathrm{H}$ NMR: $\delta 9.05 \mathrm{~s}, 1 \mathrm{H}$, $\delta 8.55 \mathrm{~s}, 2 \mathrm{H}, \delta 7.45 \mathrm{t}, 6 \mathrm{H}, \delta 7.32 \mathrm{~d}, 4 \mathrm{H}, \delta 3.65 \mathrm{~s}, 1 \mathrm{H}, \delta 3.20-2.7$ m, 4H, $\delta 2.26$ m, $1 \mathrm{H}, \delta 1.27$ t, 4H, Mass: M: 461.47, M+H: 462.1 .
Synthesis of (4-(hydroxydiphenylmethyl)piperidin-1yl)(4-bromophenyl)methanone (5c): ${ }^{1} \mathrm{H}$ NMR: $\delta 7.4-7.5 \mathrm{~m}$, $6 \mathrm{H}, \delta 7.29-7.32 \mathrm{t}, 4 \mathrm{H}, \delta 7.2 \mathrm{~m}, 4 \mathrm{H}, \delta 4.74 \mathrm{~s}, 1 \mathrm{H}, \delta 3.7 \mathrm{~s}, 1 \mathrm{H}, \delta$ $2.87 \mathrm{~s}, 1 \mathrm{H}, \delta 2.0 \mathrm{t}, 2 \mathrm{H}, \delta 2.2 \mathrm{~s}, 1 \mathrm{H}, \delta 1.27-1.58 \mathrm{~m}, 4 \mathrm{H}$. Mass: M: 450.37, M+H: 451.6.

Synthesis of (4-(hydroxydiphenylmethyl)piperidin-1yl)(4-methyl-3-nitrophenyl)-methanone (5d): ${ }^{1} \mathrm{H}$ NMR: $\delta 7.99 \mathrm{~s}, 1 \mathrm{H}, \delta 7.51 \mathrm{~m}, 10 \mathrm{H}, \delta 7.2 \mathrm{~d}, 2 \mathrm{H}, \delta 3.4-4.9 \mathrm{~m}, 2 \mathrm{H}, \delta$ 2.8 t, $2 \mathrm{H}, \delta 2.6 \mathrm{~s}, 3 \mathrm{H}, \delta$ 1.4-1.9 m, 4H. Mass: M: $430.5 \mathrm{M}+\mathrm{H}$ : 431.3 .

Synthesis of (4-(hydroxydiphenylmethyl)piperidin-1yl)(2-methyl-3-nitrophenyl)methanone (5e): ${ }^{1} \mathrm{H}$ NMR : $\delta 7.8$ $\mathrm{d}, 1 \mathrm{H}, \delta 7.45 \mathrm{~d} 2 \mathrm{H}, \delta 7.40 \mathrm{t}, 3 \mathrm{H}, \delta 7.33 \mathrm{~d}, 4 \mathrm{H}, \delta 7.2 \mathrm{~m}, 2 \mathrm{H}, \delta$ $7.17 \mathrm{t} 2 \mathrm{H}, \delta 4.85 \mathrm{t} 1 \mathrm{H}, \delta 3.36 \mathrm{~d} 1 \mathrm{H}, \delta 3.02 \mathrm{~m} 1 \mathrm{H}, \delta 2.8 \mathrm{t} 1 \mathrm{H}, \delta$ $2.65 \mathrm{~m} 1 \mathrm{H}, \delta 2.41 \mathrm{~d} 3 \mathrm{H}, \delta 2.1 \mathrm{~s} 2 \mathrm{H}, \delta 1.72 \mathrm{~d} 1 \mathrm{H}, \delta 1.53 \mathrm{~s} 2 \mathrm{H}, \delta$ $1.4 \mathrm{~m} 3 \mathrm{H}, \delta 1.29 \mathrm{~m} 1 \mathrm{H}$, Mass: M: 430.5, M+H: 431.

Synthesis of (4-(hydroxyl diphenyl methyl)piperidin1-yl)-(4-methylphenyl)methanone (5f): ${ }^{1} \mathrm{H}$ NMR: $\delta 7.44 \mathrm{~d}$, $1 \mathrm{H}, \delta 7.29 \mathrm{~m}, 6 \mathrm{H}, \delta 7.18 \mathrm{~d}, 4 \mathrm{H}, \delta 4.77 \mathrm{~s}, 1 \mathrm{H}, \delta 3.82 \mathrm{~s}, 1 \mathrm{H}, \delta$ 2.26-3.03 m, 3H, $\delta 2.36 \mathrm{~s}, 3 \mathrm{H}, \delta 1.25-1.70 \mathrm{~m}, 5 \mathrm{H}$. Mass: $\mathrm{M}^{+}$: 385.5, M+H: 386.8 .

Synthesis of (4-(hydroxyl diphenyl methyl)piperidin1-yl)(3, 5-dimethylphenyl)methanone (5g): ${ }^{1} \mathrm{H}$ NMR: $\delta 7.45$ $\mathrm{d}, 4 \mathrm{H}, \delta 7.30 \mathrm{t}, 4 \mathrm{H}, \delta 7.19 \mathrm{t}, 2 \mathrm{H}, \delta 6.98 \mathrm{~s}, 1 \mathrm{H}, \delta 6.95 \mathrm{~s}, 2 \mathrm{H}, \delta$ $4.76 \mathrm{~s}, 1 \mathrm{H}, \delta 2.88 \mathrm{bs}, 2 \mathrm{H}, \delta 2.69 \mathrm{t}, 1 \mathrm{H}, \delta 2.29 \mathrm{~s}, 1 \mathrm{H}, \delta 1.27 \mathrm{~s}$, $6 \mathrm{H}, \delta 2.11 \mathrm{t}, 1 \mathrm{H}, \delta 1.43 \mathrm{t}, 3 \mathrm{H}$. Mass: $\mathrm{M}^{+}: 399.52, \mathrm{M}+\mathrm{H}: 400.5$.

Cup-Plate method: The antimicrobial activity of synthesized analogues was tested by cup and plate method [17], Nutrient agar was poured onto the sterilized petri-dishes (20$25 \mathrm{~mL}$ each pertri dish). The poured material was allowed to set (1-1.5 h) and thereafter the "Cup-Plate method" (10 mm diameter) were made by punching into the agar surface with a sterile cork borer and scooping out the punched part of the agar. Into these cups, the test compound solution was added with the help of sterile syringe. The plates were incubated at $37^{\circ} \mathrm{C}$ for $48 \mathrm{~h}$ and the results were noted. A solvent control (10\% DMSO in methanol) was also run to not the activity of the blank (solvent). The standard drug fexofenadine was also screened under similar conditions for comparison.

\section{RESULTS AND DISCUSSION}

Four drugs has been synthesized using piperidine moiety as a common scaffold and exhibiting their perfect drug activity at various levels of absorbance, if you see none of the drug was constructed with peptide bond (-CO-NH-) at secondary amine junction of piperidine ring to form tertiary amine.

To increase the drug activity of piperidine tertiary amine functional group, it is thought to construct a peptide bond formation with secondary amine of piperidine by treating with some substituted aromatic carbonyl chlorides in presence of a base often to form some biologically active pipradol derivatives.

The synthesized analogues of (4-(hydroxyl diphenyl methyl)piperidin-1-yl) (substituted phenyl)methanone from commercially available diphenyl(piperidin-4-yl)methanol (azacyclonol) were examined for its biological activity and the obtained results were showing more potential towards antimicrobial activity against Candida albicans, Aspergillus niger and Saccharomyces cerrevisiae (Table-1). 


\begin{tabular}{|c|c|c|c|c|c|c|c|c|}
\hline \multirow{3}{*}{$\begin{array}{l}\text { Conc. } \\
(\mu \mathrm{g} / \mathrm{mL})\end{array}$} & \multicolumn{7}{|c|}{$\begin{array}{l}\text { TABLE-1 } \\
\text { MICROBIOLOGY TESTING RESULTS; STD: FEXOFENADINE DRUG STANDARD; } \\
\mathbf{5 a}, \mathbf{5 b}, \mathbf{5 c}, \mathbf{5 d}, \mathbf{5 e}, \mathbf{5 f}, \mathbf{5 g} \text { ARE SYNTHESIZED DERIVATIVES }\end{array}$} & \\
\hline & \multicolumn{8}{|c|}{ Microbiology - Cell cultures } \\
\hline & $5 \mathbf{5 a}$ & $\mathbf{5 b}$ & $5 \mathbf{c}$ & $5 d$ & $5 e$ & $5 f$ & $5 g$ & Std \\
\hline \multicolumn{9}{|c|}{ Aspergillums niger } \\
\hline 1.0 & 10.0 & 10.1 & 10.1 & 10.1 & 10.0 & 10.1 & 10.0 & 09.9 \\
\hline 2.0 & 10.2 & 10.2 & 10.2 & 10.1 & 10.1 & 10.1 & 10.0 & 10.1 \\
\hline 3.0 & 10.4 & 10.2 & 10.2 & 10.1 & 10.0 & 10.1 & 10.0 & 10.1 \\
\hline 4.0 & 10.5 & 10.2 & 10.3 & 10.1 & 10.1 & 10.1 & 10.0 & 10.2 \\
\hline 5.0 & 10.5 & 10.2 & 10.3 & 10.2 & 10.1 & 10.1 & 10.0 & 10.1 \\
\hline 6.0 & 10.4 & 10.4 & 10.3 & 10.4 & 10.1 & 10.3 & 10.2 & 10.4 \\
\hline 7.0 & 10.6 & 10.4 & 10.4 & 10.4 & 10.1 & 10.4 & 10.2 & 10.4 \\
\hline 8.0 & 10.8 & 10.5 & 10.5 & 10.5 & 10.1 & 10.4 & 10.4 & 10.4 \\
\hline 9.0 & 10.8 & 10.5 & 10.5 & 10.5 & 10.3 & 10.4 & 10.6 & 10.7 \\
\hline \multicolumn{9}{|c|}{ Candida albicans } \\
\hline 1.0 & - & - & - & - & - & - & 10.1 & - \\
\hline 2.0 & - & - & - & - & - & - & 10.1 & 10.2 \\
\hline 3.0 & - & - & - & - & - & - & 10.1 & 10.5 \\
\hline 4.0 & - & - & - & - & - & - & 10.3 & 10.6 \\
\hline 5.0 & - & - & - & - & - & - & 10.3 & 10.8 \\
\hline 6.0 & - & - & - & - & - & - & 10.3 & 10.8 \\
\hline 7.0 & - & - & - & - & - & - & 10.3 & 10.9 \\
\hline 8.0 & - & - & 10.2 & - & - & - & 10.3 & 10.8 \\
\hline 9.0 & - & - & - & - & - & - & 10.5 & 10.9 \\
\hline \multicolumn{9}{|c|}{ Saccharomyces cerevisiae } \\
\hline 1.0 & - & - & - & 11.1 & - & - & - & - \\
\hline 2.0 & - & - & - & 11.2 & - & - & - & 09.6 \\
\hline 3.0 & - & 10.4 & - & 11.2 & - & - & 11.3 & 09.6 \\
\hline 4.0 & 11.1 & 10.7 & - & 11.3 & - & - & 11.8 & 09.8 \\
\hline 5.0 & 11.0 & 10.8 & - & 11.3 & - & - & 11.8 & 09.9 \\
\hline 6.0 & 11.1 & 10.8 & 11.4 & 11.3 & - & - & 11.9 & 09.9 \\
\hline 7.0 & 11.2 & 11.4 & 11.5 & 11.3 & - & - & 11.9 & 10.3 \\
\hline 8.0 & 11.2 & 11.9 & 11.5 & 11.4 & - & - & 11.9 & 10.5 \\
\hline 9.0 & 11.7 & 11.9 & 11.7 & 11.6 & - & - & 11.9 & 10.8 \\
\hline
\end{tabular}

\section{Conclusion}

A new attempt is made for synthesizing a potential 4(hydroxyldiphenyl methyl)piperidin-1-yl)(substituted phenyl)methanone derivatives as antimicrobial agent. Among the six synthezied compounds, more or less all are gets positive range of activity against fexofenadine, but compounds $\mathbf{5 g}, \mathbf{5 c}$ and $\mathbf{5 d}$ were seems to be more attractive and promising molecules for extended research work, which can be use full to identify a new drug product for drug therapy applications.

\section{ACKNOWLEDGEMENTS}

Our first and foremost thanks to CSIR for providing the fellowship, then for Department of Chemistry of Acharya Nagarjuna University and M/s Srigen Life Sciences Pvt. Ltd. for providing the laboratory facility to do its part of the work.

\section{REFERENCES}

1. M. Pieroni, D. Machado, E. Azzali, S. Santos Costa, I. Couto, G. Costantino and M. Viveiros, J. Med. Chem., 58, 5842 (2015); https://doi.org/10.1021/acs.jmedchem.5b00428.

2. R.C. Krauss, R.M. Strom, C.L. Scortichini, W.J. Kruper, R.A. Wolf, W.W. Wu, A.A. Carr, D.A. Hay, D.E. Rudisill and G.P. Weniger, Intermediates Useful for the Preparation of Antihistaminic Piperidine Derivatives, US Patent 6555689 B2 (2003).

3. M.G. Van Campen Jr , C.P. Raymond and L.S. Edward, Tranquilizing Composition Comprising Alpha phenyl, Alpha-(4-piperidyl)benzyl Alcohol, US Patent 2804422 A (1957).
4. F.J. McCarty, C.H. Tilford and M.G. Van Campen Jr., J. Am. Chem. Soc., 79, 472 (1957); https://doi.org/10.1021/ja01559a066.

5. E. Franklin, J. Pharmacol. Exp. Ther., 124, 347 (1958).

6. F.E. Roth, Chemotherapia, 3, 120 (1961); https://doi.org/10.1159/000219537.

7. L.D. Simmler, A. Rickli, Y. Schramm, M.C. Hoener and M.E. Liechti, Biochem. Pharmacol., 88, 237 (2014); https://doi.org/10.1016/j.bcp.2014.01.024.

8. S.H. Kawai, R.J. Hambalek and G. Just, J. Org. Chem., 59, 2620 (1994); https://doi.org/10.1021/jo00088a056.

9. J.K. Woodward and N.L. Munro, Arzneimittelforschung, 32, 1154 (1982).

10. Merck Index, Monograph no. 9094, p. 1443, edn 11, (1989).

11. E. Thomas and D. Ambra, Piperidine Serivatives and Process for their Preparation, US Patent 5750703 A (1998).

12. D. Zivkovic and R.-S. Genese, N-Hydroxyalkyl, Hydroxyalkoxyalkyl and Hydroxyalkoxyalkoxy-alkyl diphenyl-methyl Piperidine, US Patent 3687956 A (1972).

13. W. Ronggeng, Z. Yougui and Z. Guanchao, Res. Chem. Intermed., 39, 2149 (2013);

https://doi.org/10.1007/s11164-012-0747-y.

14. Fine Spec. Chem., 19, 32 (2011).

15. M. Li, G. Mengyu, C. Fengqiang, The synthase of the intermediates for fexofenadine CN 101182306A.

16. K. Hoffmann and J. Heer, Process for the manufacture of second-Ary Diphenyl-piperldyl-carbinols, US Patent 3,000,896 (1961).

17. R. Cruickshank, J.P. Duguid, B.P. Marmion and R.H.A. Swain, The enterobacteriaceae: Salmonella; In: Medical Microbiology, Churchill Livingstone, Edinburgh, London and New York, vol. 11, edn 12, pp. 403-419 (1975). 